\title{
Universiteit
}

Leiden

The Netherlands

\section{Frequency control of photonic crystal membrane resonators by mono- layer deposition}

Strauf, S.; Rakher, M.T.; Carmeli, I.; Hennessy, K.T.; Meier, C.; Badolato, A.; ... ;

Bouwmeester, D.

\section{Citation}

Strauf, S., Rakher, M. T., Carmeli, I., Hennessy, K. T., Meier, C., Badolato, A., ...

Bouwmeester, D. (2006). Frequency control of photonic crystal membrane resonators by mono-layer deposition. Applied Physics Letters, 88, 043116. doi:10.1063/1.2164922

Version: $\quad$ Not Applicable (or Unknown)

License: $\quad$ Leiden University Non-exclusive license

Downloaded from: https://hdl.handle.net/1887/65882

Note: To cite this publication please use the final published version (if applicable). 


\section{Frequency control of photonic crystal membrane resonators by monolayer deposition}

S. Strauf, M. T. Rakher, I. Carmeli, K. Hennessy, C. Meier, A. Badolato, M. J. A. DeDood, P. M. Petroff, E. L. Hu, E. G. Gwinn, and D. Bouwmeester

Citation: Appl. Phys. Lett. 88, 043116 (2006); doi: 10.1063/1.2164922

View online: https://doi.org/10.1063/1.2164922

View Table of Contents: http://aip.scitation.org/toc/apl/88/4

Published by the American Institute of Physics

\section{Articles you may be interested in}

Scanning a photonic crystal slab nanocavity by condensation of xenon

Applied Physics Letters 87, 141105 (2005); 10.1063/1.2076435

Invited Review Article: Single-photon sources and detectors

Review of Scientific Instruments 82, 071101 (2011); 10.1063/1.3610677

High quality factor photonic crystal nanobeam cavities

Applied Physics Letters 94, 121106 (2009); 10.1063/1.3107263

Tuning photonic crystal nanocavity modes by wet chemical digital etching

Applied Physics Letters 87, 021108 (2005); 10.1063/1.1992656

Cavity-quantum electrodynamics using a single InAs quantum dot in a microdisk structure

Applied Physics Letters 78, 3932 (2001); 10.1063/1.1379987

Achievement of ultrahigh quality factors in GaAs photonic crystal membrane nanocavity

Applied Physics Letters 89, 221104 (2006); 10.1063/1.2390648

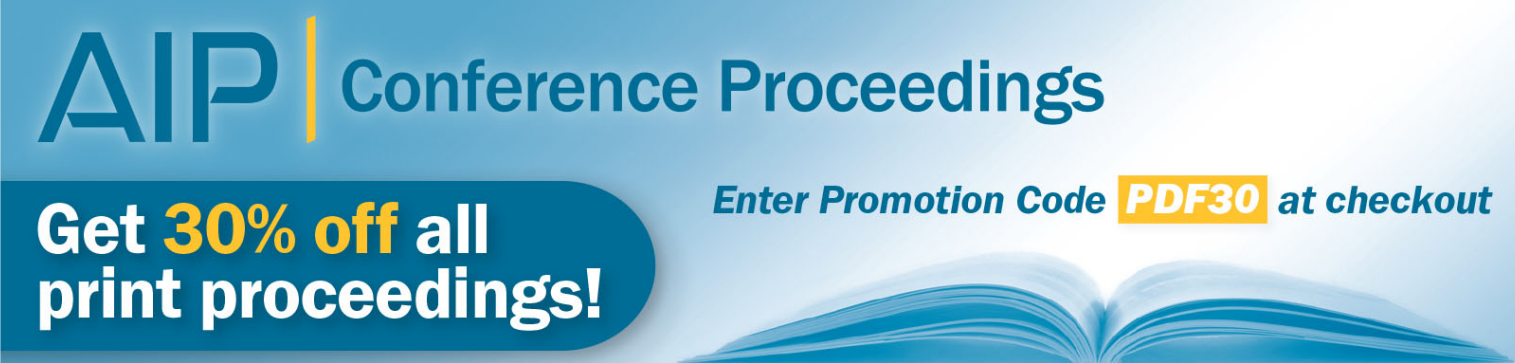




\title{
Frequency control of photonic crystal membrane resonators by monolayer deposition
}

\author{
S. Strauf, ${ }^{\text {a),b) }}$ M. T. Rakher, ${ }^{\text {b) }}$ I. Carmeli, ${ }^{\text {b) }}$ K. Hennessy, ${ }^{\text {c) }}$ C. Meier, ${ }^{\text {c),d) }}$ A. Badolato, ${ }^{\text {c) }}$ \\ M. J. A. DeDood, ${ }^{\text {b) }}$ P. M. Petroff, ${ }^{c), e)}$ E. L. Hu, ${ }^{\text {c),e) }}$ E. G. Gwinn, ${ }^{\text {b) }}$ and D. Bouwmeester ${ }^{\text {b) }}$ \\ University of California, Santa Barbara, Santa Barbara, California 93106
}

(Received 15 August 2005; accepted 18 November 2005; published online 27 January 2006)

\begin{abstract}
We study the response of GaAs photonic crystal membrane resonators to thin-film deposition. Slow spectral shifts of the cavity mode of several nanometers are observed at low temperatures, caused by cryo-gettering of background molecules. Heating the membrane resets the drift and shielding will prevent drift altogether. In order to explore the drift as a tool to detect surface layers, or to intentionally shift the cavity resonance frequency, we studied the effect of self-assembled monolayers of polypeptide molecules attached to the membranes. The 2-nm-thick monolayers lead to a discrete step in the resonance frequency and partially passivate the surface. (C) 2006 American Institute of Physics. [DOI: 10.1063/1.2164922]
\end{abstract}

Photonic crystal membrane microcavities (PCMs) are promising candidates for applications ranging from quantum and classical communication, ${ }^{1}$ to microlasers ${ }^{2-4}$ and sensing devices. ${ }^{5,6}$ Due to their ultrasmall mode volumes ${ }^{7}$ and large surface to volume ratio, the PCMs' resonant frequency is highly sensitive to its environment. While this sensitivity may be exploited for novel sensing applications, it complicates solid-state cavity quantum electrodynamic (QED) experiments that depend on a precise resonance condition between a cavity mode and an embedded single quantum dot (QD), ${ }^{8-11}$ single atom ${ }^{12}$ or single impurity. ${ }^{13}$ This letter describes a slow redshift of the PCM mode emission frequency that can occur at low operation temperatures. We ascribe this shift to molecular condensation on the PCM surface. We further describe methods used to fully curtail the drift, and in addition, we report on the first demonstration of a controlled redshift of the PCM mode by the adsorption of a selfassembled monolayer (SAM) of polypeptide molecules.

We are particularly interested in PCM devices operated at low temperatures in such a way that embedded QDs display a discrete energy spectrum. As a model system a squarelattice PCM geometry with one missing air hole (S1) has been chosen, which is known to confine the fundamental mode in the proximity of the air-semiconductor interface. ${ }^{14} \mathrm{~A}$ single layer of self-assembled InAs QDs was embedded in the 180-nm-thick GaAs membrane and emits around $950 \mathrm{~nm}^{15}$ under nonresonant laser excitation at $780 \mathrm{~nm}$. Figure 1(a) shows a spectrum of the fundamental cavity mode taken at pump powers of $15 \mu \mathrm{W}$, which has been recorded with a microphotoluminescence (micro-PL) setup. ${ }^{16}$ At these excitation conditions the cavity mode is clearly visible at $1.3293 \mathrm{eV}$ with a quality factor ( $Q$ factor) of 1900 . The $2-\mu \mathrm{m}$-diam laser excitation spot has to be positioned with an accuracy of $\pm 0.5 \mu \mathrm{m}$ with respect to the cavity defect region [Fig. 1(c)], demonstrating the strongly localized character of

\footnotetext{
a)Author to whom correspondence should be addressed; electronic mail: strauf@physics.ucsb.edu

${ }^{b}$ Physics Department.

${ }^{c}$ Electrical Engineerig Department.

${ }^{d)}$ Present address: Institute for Experimental Physics, University of Duisburg-Essen, D-47048 Duisburg, Germany.

${ }^{\text {e) }}$ Materials Department.
}

the mode. Individual QD transitions are visible under low pump power excitation of $50 \mathrm{nW}$, which have been identified by their pronounced antibunching signature (not shown). Another set of two spectra was taken 200 min later as shown in Fig. 1(b). While the single QD emission energy at $1.31780 \pm 2 \cdot 10^{-5} \mathrm{eV}$ did not change in the entire observation period, the cavity mode has now redshifted by $5.8 \mathrm{meV}$ $(4 \mathrm{~nm})$ and has a slightly lower $Q$ factor of 1700 . The energetically stable QD emission indicates that no temperature drift or strain-induced modification of the electronic states ${ }^{17}$ occurred over time.

The cavity mode energy shift as a function of time is shown in Fig. 2(a). The observed redshift slows down and saturates after a few hours. Measurements on different samples show that the drift is mostly independent of the actual $r / a$ ratio of the $\mathrm{S} 1$ cavities. It is known that chemical wet etching of the PCM structures in HF and selective removal of a self-formed native oxide will result in a systematic blueshift of the cavity mode. ${ }^{14}$ Therefore, we believe that the measured redshift can be ascribed to material being added (adsorbed) onto the same surface through cryo-gettering. ${ }^{18}$ In confirmation of this hypothesis, we have

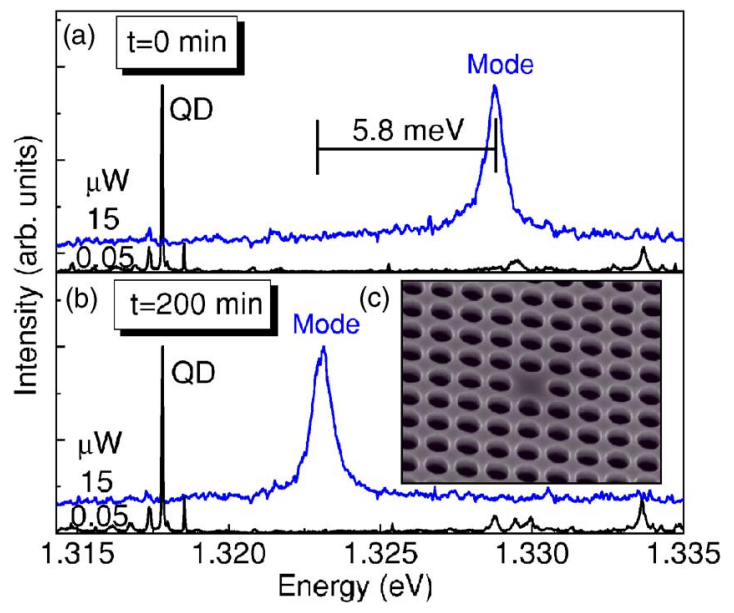

FIG. 1. (Color online) (a) Drift of the cavity mode energy with time. Cycling the temperature from 4 to $410 \mathrm{~K}$ and back to $4 \mathrm{~K}$ resets the mode back to $1.3295 \mathrm{eV}$ and the drift starts again as shown in Fig. 2(b). Data are taken at $4.2 \mathrm{~K}$ and $15 \mu \mathrm{W}$ pump power. 


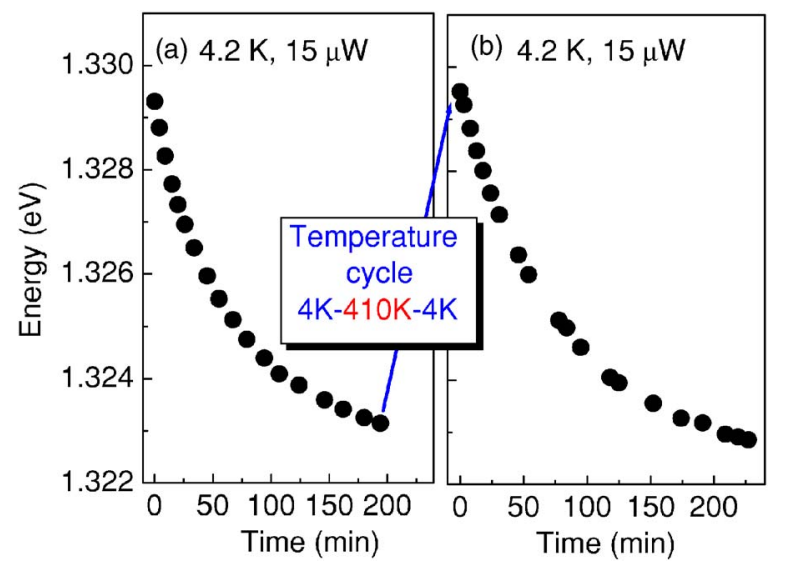

FIG. 2. (Color online) Low temperature (4.2 K) micro-PL spectra of a PCM shortly after cooldown (a) and 200 min later (b). High pump power spectra $(15 \mu \mathrm{W}$, blue) show the cavity mode and low pump power spectra $(0.05 \mu \mathrm{W}$, black) show a single QD emitting at $1.318 \mathrm{eV}$. (c) Scanning electron micrograph of a PCM with a lattice constant $a$ of $290 \mathrm{~nm}$ and hole radius $r$ of $110 \mathrm{~nm}$ around the defect region.

found that the redshift of the cavity mode can be fully recovered by cycling the sample temperature from 4 to $410 \mathrm{~K}$ and back to $4 \mathrm{~K}$ [Fig. 2(b)], demonstrating that the thin film can be fully removed.

A more detailed study reveals that the temperature dependence of the mode shift is varying as shown in Fig. 3(a). All temperature data have been recorded $5 \mathrm{~h}$ after initial cooldown to $4 \mathrm{~K}$ in the regime where the mode drift over time is saturated. With increasing temperature the mode energy is nearly constant between 4 and $30 \mathrm{~K}$, blueshifts by $1 \mathrm{~nm}$ between 30 and $50 \mathrm{~K}$ and is followed by a redshift of $2 \mathrm{~nm}$ up to $100 \mathrm{~K}$ (solid dots). In contrast, the single QD emission energy [Fig. 3(b), crosses] follows the expected temperature dependence of the semiconductor band gap [Fig. 3 (b), red line], according to the Bose-Einstein model ${ }^{19}$ with parameters as given in the inset. This demonstrates that the actual temperature readout of the $\mathrm{Si}$ thermometer inside the cryostat is close to the real sample temperature within the PCM defect region, where the single QD is located. For temperatures above $50 \mathrm{~K}$ the slope of the mode energy follows the expected linear redshift due to the temperature dependence of the effective refractive index $n(T)$, as has been cal-
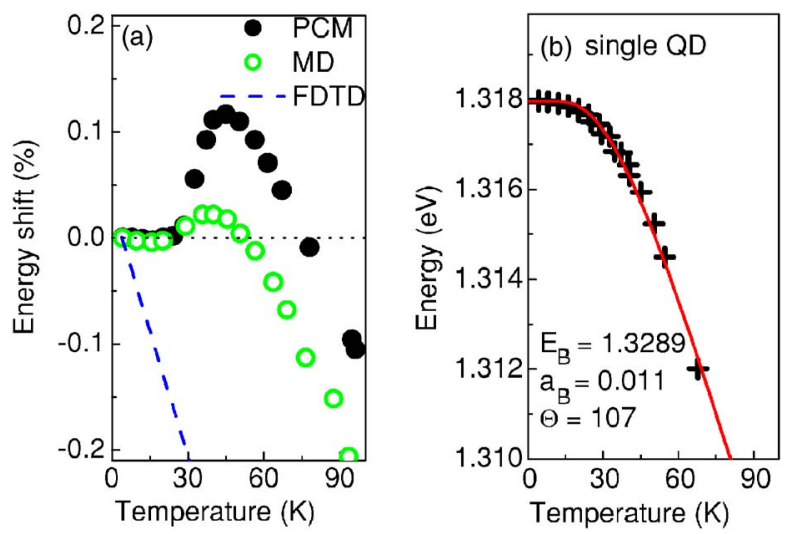

FIG. 3. (Color online) (a) Temperature dependence of the mode emission energy for a PCM (solid dots) and a MD (open dots). Dashed line: finitedifference time-domain calculation of the mode shift with temperature for a PCM. (b) Temperature dependence of the single QD transition energy (crosses). The red line is a fit according to the Bose-Einstein model. Mode energies are normalized to the $T=4 \mathrm{~K}$ values.

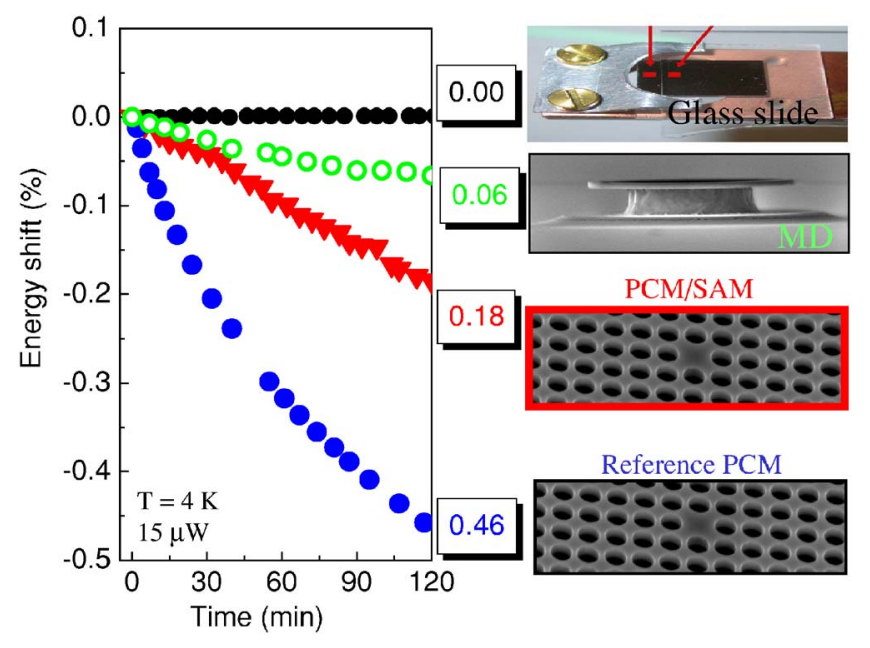

FIG. 4. (Color online) Comparison of cavity mode redshift over time for a reference PCM (blue dots), a SAM-coated PCM (red triangles), a microdisk (open green dots) and a PCM capped with a thin glass slide (black dots). Mode energies are normalized to the $t=0 \mathrm{~min}$ values. Data are taken at $4.2 \mathrm{~K}$ and $15 \mu \mathrm{W}$ pump power.

culated by finite-difference time-domain simulations (dashed line) assuming that $n(T)$ of the GaAs membrane changes with temperature according to Ref. 20. Thus, the observed anomalous blueshift at temperatures around $40 \mathrm{~K}$ must be caused by an additional effect and is attributed to a partial desorption and/or reconfiguration of the deposited film.

By way of comparison, we studied the behavior of the whispering-gallery mode (WGM) of microdisk (MD) structures with $5 \mu \mathrm{m}$ diameter, which have been defined by optical lithography and transferred into the GaAs by a two-step wet etch process based on $\mathrm{HBr}^{21}$ These devices show a largely reduced blueshift with increasing temperature [Fig. 3(a) open dots] as well as a largely reduced frequency shift over time [Fig. 4, open green dots] compared to PCM reference devices (Fig. 4, blue dots). The WGMs of the MD structures have some evanescent coupling in both lateral and vertical direction, but the PCM mode penetrates much further into the air-hole region ${ }^{14}$ and is therefore more susceptible to the environment.

On the one hand the pronounced sensitivity of the PCM mode to the actual environmental conditions is promising for chemical sensing applications. On the other hand, this sensitivity might significantly complicate the analysis of cavityQED experiments utilizing temperature tuning in order to establish a resonance condition between the cavity mode and the emission energy of an embedded single quantum dot. In order to fully stop the mode energy drift over time the PCM devices have been capped with a thin glass slide. ${ }^{22}$ As a result, the energy drift is now completely absent (Fig. 4 black dots). While this approach is satisfactory for cavity-QED experiments at low temperatures, it would be highly desirable to directly manipulate the PCM surface, allowing for selective sensing of chemical species and to interface with functional molecules and/or colloidal QDs. To this end we linked polypeptide SAMs to the GaAs surface of the PCM. The polypeptide molecule is composed of eight alternating (AlaAib) sequences with a Glutamic acid (Glu) attached to the C terminal of the peptide and is stable in $\alpha$-helix form. ${ }^{23}$ The peptide chemically binds to the GaAs surface by a carboxyl group located in the Glu amino acid. It was adsorbed to the GaAs surface by first removing the surface oxide in dilute 
$\mathrm{HF}$, and then immediately immersing it in $1 \mathrm{mM}$ solution of the molecule in absolute ethanol.

The thickness of the monolayer was measured by ellipsometry to be $2.2 \pm 0.2 \mathrm{~nm}$, in agreement with the calculated length of the polypeptide of $2.6 \mathrm{~nm}$. This indicates that the molecules have formed a monolayer with the long molecule axis almost perpendicular to the surface. Samples with an attached SAM and initially capped with a glass slide show a redshifted PCM mode by about 3-5 nm compared to reference samples without SAMs. This indicates that the SAM is indeed attached to the GaAs surface and highlights the pronounced sensitivity of the S1 cavity mode's ability to sense layers only $2 \mathrm{~nm}$ thick with a frequency response about 6-10 times larger than the full width at half maximum of the cavity mode $(0.5 \mathrm{~nm})$. This sensitivity can be further increased by use of $\mathrm{S} 1$ cavities with demonstrated $Q$ factors as high as $10000 .^{14}$

Finally, we removed the glass slide from the SAM covered sample and found a largely reduced magnitude of the cavity mode redshift over time by up to a factor of 3 (Fig. 4, red triangles) compared to untreated PCM reference devices (Fig. 4, blue dots). This demonstrates a partial surface passivation once a SAM is attached. It is furthermore expected that the use of molecules with longer chains and thus larger average film thickness would give rise to a further reduction of the mode shift over time.

In summary, our experiments show how one may control the environment of a PCM to obtain either stable, stepped or continuous tuning operation, each of which will be of interest in a variety of nanophotonic applications. We demonstrated a new method to attach self-assembled monolayers to GaAs photonic crystal membrane cavities opening novel possibilities for biofunctionalized photonic devices.

This research has been supported through NSF NIRT Grant No. 0304678 and DARPA Grant No. MDA 972-01-10027. We acknowledge Atac Imamoglu and Mete Atatüre for fruitful discussions.

${ }^{1}$ D. Bouwmeester, A. Ekert, and A. Zeilinger, The Physics of Quantum Information Technology (Springer, New York, 2000).

${ }^{2}$ O. Painter, R. K. Lee, A. Scherer, A. Yariv, J. D. O’Brien, P. D. Dapkus, and I. Kim, Science 284, 1819 (1999).

${ }^{3}$ H.-G. Park, Se-Heon Kim, Soon-Hong Kwon, Young-Gu Ju, Jin-Kyu Yang, Jong-Hwa Baek, Sung-Bock Kim, and Yong-Hee Lee, Science 305, 1444 (2004).

${ }^{4}$ S. Strauf, K. Hennessy, M. T. Rakher, Y.-S. Choi, A. Badolato, L. C. Andreani, E. L. Hu, P. M. Petroff, and D. Bouwmeester, submitted to Phys. Rev. Lett., preprint available at http://arxiv.org/abs/cond-mat/ 0511494

${ }^{5}$ M. Loncar, A. Scherer, and Y. Qiu, Appl. Phys. Lett. 84, 4648 (2003).

${ }^{6}$ E. Chow, A. Grot, L. W. Mirkarimi, M. Sigales, and G. Girolami, Opt. Lett. 29, 1093 (2004).

${ }^{7}$ K. J. Vahala, Nature (London) 424, 839 (2003).

${ }^{8}$ P. Michler, A. Kiraz, C. Becher, W. V Schoenfeld, P. M. Petroff, L. Zhang, E. Hu, and A. Imamoglu, Science 290, 2282 (2000).

${ }^{9}$ J. Vuckovic, D. Fattal, C. Santori, G. S. Solomon, and Y. Yamamoto, Appl. Phys. Lett. 82, 3596 (2003).

${ }^{10}$ J. P. Reithmeier, G. Sek, A. Löffler, C. Hofmann, S. Kuhn, S. Reitzenstein, L. V. Keldysh, V. D. Kulakovskii, T. L. Reinecke, and A. Forchel, Nature (London) 432, 197 (2004).

${ }^{11}$ T. Yoshie, A. Scherer, J. Hendrickson, G. Khitrova, H. M. Gibbs, G. Rupper, C.. Ell, O. B. Shchekin, and D. G. Deppe, Nature (London) 432, 200 (2004).

${ }^{12}$ J. Vuckovic, M. Loncar, H. Mabuchi, and A. Scherer, Phys. Rev. E 65, 016608 (2002).

${ }^{13}$ S. Strauf, P. Michler, M. Klude, D. Hommel, G. Bacher, and A. Forchel, Phys. Rev. Lett. 89, 177403 (2002).

${ }^{14}$ K. Hennessy, A. Badolato, A. Tamboli, P. M. Petroff, E. Hu, M. Atatüre, J. Dreiser, and A. Imamoglu, Appl. Phys. Lett. 87, 021108 (2005).

${ }^{15}$ J. M. Garcia, P. Mankad, P. O. Holtz, P. J. Wellman, and P. M. Petroff, Appl. Phys. Lett. 72, 3172 (1998).

${ }^{16}$ B. D. Gerardot, S. Strauf, J. M. A. DeDood, A. M. Bychkov, A. Badolato, K. Hennessy, E. L. Hu, D. Bouwmeester, and P. M. Petroff, Phys. Rev. Lett. 95, 137403 (2005).

${ }^{17}$ T. Nakaoka, T. Kakitsuka, T. Saito, and Y. Arakawa, Appl. Phys. Lett. 84, 1392 (2004).

${ }^{18} \mathrm{~A}$ similar drift of the PCM mode energy has been observed in four different optical cryostates (Oxford), although the vacuum was better than $1 \times 10^{-6}$ mTorr.

${ }^{19}$ E. Grilli, M. Guzzi, R. Zamboni, and L. Pavesi, Phys. Rev. B 45, 1638 (1992).

${ }^{20}$ D. C. Reynolds, K. K. Bajaj, C. W Litton, G. Peters, P. W. Yu, and J. D. Parsons, J. Appl. Phys. 61, 342 (1987).

${ }^{21}$ P. Michler, A. Kiraz, L. Zang, C. Becher, and A. Imamoglu, Appl. Phys. Lett. 77, 184 (2000).

${ }^{22}$ The barely visible film gettered on the glass slide dissolves completely in acetone but not in de-ionized water, indicating that the material is hydrocarbon related.

${ }^{23}$ Y. Miura, S. Kimura, Y. Imanishi, and J. Umemura, Langmuir 14, 6935 (1998). 